\title{
The effect of lithium carbonate on angiogenesis of hepatocellular carcinoma-29
}

\author{
Viktoriia Makarova \\ Laboratory of ultrastructural research, \\ Research Institute of Clinical and \\ Experimental Lymphology - Branch of \\ the Institute of Cytology and Genetics, \\ Siberian Branch of the Russian \\ Academy of Sciences \\ Novosibirsk, Russia \\ shedina_vika@mail.ru
}

\author{
Nataliya Bgatova \\ Laboratory of ultrastructural research, \\ Research Institute of Clinical and \\ Experimental Lymphology - Branch of \\ the Institute of Cytology and Genetics, \\ Siberian Branch of the Russian \\ Academy of Sciences \\ Novosibirsk, Russia \\ n_bgatova@ngs.ru
}

\author{
Iuliia Taskaeva \\ Laboratory of ultrastructural research, \\ Research Institute of Clinical and \\ Experimental Lymphology - Branch of \\ the Institute of Cytology and Genetics, \\ Siberian Branch of the Russian \\ Academy of Sciences \\ Novosibirsk, Russia \\ inabrite@yandex.ru
}

\begin{abstract}
Hepatocellular carcinoma is one of the most aggressive human tumors with a high prevalence and mortality rate. At the same time, there is a problem of $\mathrm{HCC}$ resistance to standard therapy. The search for drugs that block the growth and metastasis of the tumor is relevant. There is evidence of antitumor properties of lithium carbonate. The aim of our study was to determine the effect of lithium on the development and structure of blood vessels of experimental hepatocellular carcinoma-29 (G-29). The study was performed on male CBA mice. Hepatocarcinoma-29 cells were used to induce the tumor process. The expression of the blood vessel endothelial marker (CD-31) and the ultrastructural organization of tumor endothelial cell vessels were studied. It was shown that lithium carbonate decrease volume density of blood vessels G-29. Ultrastructural analysis of the tumor vessels revealed the atypical nature of their structure. The walls of the vessels were lined with both endothelial and tumor cells.
\end{abstract}

Keyword s- hepatocellular carcinoma, vasculogenic mimicry, angiogenesis, lithium

\section{INTRODUCTION}

Hepatocellular carcinoma is one of the most aggressive tumors with high mortality $[1,2]$. Neoangiogenesis is known to contribute to cancer progression, relapse, and metastasis. But most modern drugs aimed at inhibiting angiogenesis are ineffective [3]. Recent studies have shown that in highly aggressive tumors not only endothelial cells can be used to create vascular structures, but also alternative ways of forming the vasculature, in which vascular walls are formed by tumor cells. This is the so-called process of vasculogenic mimicry [4]. Therefore, it remains relevant to study the mechanisms of blocking vascular structures that provide trophic support for tumor growth and ways of its metastasis. There are various ways of influencing lithium on many intracellular processes, including in tumor cells $[5,6]$. For example, by inhibiting regulatory GSK3 $\beta$, induce apoptosis of cancer cells and thereby inhibit tumor growth [7]. Based on this, it is proposed to use lithium as an antitumor agent. The aim of our study was to study the effect of lithium carbonate on the development and structure of the vessels of experimental hepatocellular carcinoma-29.

\section{METHODS AND ALGORITHMS}

The study was conducted on male mice of the CBA line. Work with animals was carried out in accordance with the "Rules for the work using experimental animals." G-29 cells were used to induce the tumor process. GK-29 was obtained and verified by the Institute of Cytology and Genetics SB RAS. A suspension of GK-29 cells was injected into $0.1 \mathrm{ml}$ of intact animals into the muscle of the right thigh (3). The animals were divided into 3 groups: I - intact animals, 2 animals with tumor growth, 3 - animals with GK-29, treated with lithium carbonate as a treatment. The material for research was collected on the 30th day of the experiment. Animals were removed from the experiment under ether anesthesia using the cranio-cervical dislocation method. Tumor fragments were fixed in formalin and processed according to standard methods to obtain paraffin blocks. Paraffin sections were dewaxed, rehydrated and washed with distilled water. Antibodies anti-CD31antibody (Abcam, UK) were used to determine vascular endothelial cells. Histological preparations were stained with Mayer hematoxylin. Evaluation of the results was carried out using a light microscope (LEICA DME, Germany), with a 400-fold increase. Photos were obtained using the Avigion computer program. For electron microscopic examination, tumor tissue samples were fixed in a $1 \%$ solution of $\mathrm{OsO} 4$ in phosphate buffer $(\mathrm{pH}=7.4)$, dehydrated in increasing concentration of ethanol, and enclosed in epon (Serva, Germany). Semi-thin sections with a thickness of $1 \mu \mathrm{m}$ were stained with toluidine blue and studied under a light microscope (LEICA DME, Germany). Ultrathin sections with a thickness of 70-100 nm were contrasted with a saturated aqueous solution of uranyl acetate and lead citrate and studied using a JEM 1010 electron microscope (JEOL, Japan). Morphometric analysis was performed using Image J software (Wayne Rasband, USA).

\section{RESULTS}

Histological analysis of tumor tissue revealed a high density of vascular structures with narrow lumens. It was found that the volume density of blood vessels stained on a blood vessel marker CD-31 was significantly lower than the total volume density of all vascular structures. Ultrastructural analysis showed that part of the walls of such vessels is lined not only with endothelial cells, but also with G-29 cells. It was difficult to differentiate such vessels since they had a structure similar to both blood and lymph vessels. This phenomenon is defined as vasculogenic mimicry, which is widespread in aggressive tumors and is a poor prognostic indicator of patient survival $[8,9]$.

In the group of animals that were injected with lithium carbonate, a significant decrease in the volume density of vascular structures was noted. At the same time, the density of CD-31-positive vessels decreased to a greater extent. 
Apparently, lithium carbonate blocks the angiogenesis of true blood vessels.

\section{CONCLUSION}

The data obtained show that experimental hepatocarcinoma-29 has good vascularization. Neoangiogenesis in tumor tissue follows the path of vasculogenous mimicry, which greatly complicates the standard antitumor therapy. Lithium carbonate is a promising means of blocking tumor growth and metastasis.

\section{REFERENCES}

[1] Y. Shen, D. Cao, "Hepatocellular carcinoma stem cells: origins and roles in hepatocarcinogenesis and disease progression," Biosci., vol. 1(4), pp. 1157-1169, January 2012.

[2] B. Qu, G. Sheng, L Guo, F. Yu, G. Chen, Q. Lu, R. Wang, B. Han, Y. $\mathrm{Lu}$, "MIG7 is involved in vasculogenic mimicry formation rendering invasion and metastasis in hepatocellular carcinoma," Oncol Rep., vol. 39(2), pp. 679-686, February 2018.

[3] Y. Zeng, X. Yao, X. Liu, X. He, L. Li, X. Liu, Z. Yan, J. Wu, B.M. Fu, "Anti-angiogenesis triggers exosomes release from endothelial cells to promote tumor vasculogenesis," J Extracell Vesicles., vol. 8(1), pp. 1629865, January 2019.
[4] J. Zhang, L. Qiao, N. Liang, J. Xie, H. Luo, G. Deng, J. Zhang, "Vasculogenic mimicry and tumor metastasis," J BUON., vol. 21(3), pp. 533-541, May-June 2016.

[5] Iu. Taskaeva, N. Bgatova, I. Gogaeva, "Lithium effects on vesicular trafficking in hepatocellular carcinoma cells," Ultrastructural Pathology, vol. 43(6), pp. 301-311, 2019.

[6] Y.S. Taskaeva, N.P Bgatova, "Cytological characteristics of a heterogeneous population of hepatocellular carcinoma-29 cells after injection of lithium carbonate in the experiment," Bulletin of Experimental Biology and Medicine, vol. 167(6), pp. 779-783, 2019.

[7] J.S. Wang, C.L. Wang, J.F. Wen, Y.J. Wang, Y.B. Hu, H.Z. Ren, "Lithium inhibits proliferation of human esophageal cancer cell line Eca-109 by inducing a G2/M cell cycle arrest", World journal of gastroenterology, vol. 14, pp. 3982-3989, July 2008.

[8] M.C.M. Prado, S.A.L. Macedo, G.G. Guiraldelli, P. de Faria Lainetti, A.F. Leis-Filho, P.E. Kobayashi, R. Laufer-Amorim, C.E. FonsecaAlves, "Investigation of the Prognostic Significance of Vasculogenic Mimicry and Its Inhibition by Sorafenib in Canine Mammary Gland Tumors," Front Oncol., vol. 19(9), pp. 1445, December 2019.

[9] V.V. Makarova, N.P. Bgatova, Yu.S. Taskaeva, Yu.I., "Borodin Angiogenesis in experimental hepatocarcinoma-29," The 22nd International Charles Heidelberger Symposium on Cancer Research Proceedings of the International Symposium, pp. 67-68, 2018. 